\title{
Systematic assessment of fluid responsiveness during early septic shock resuscitation: secondary analysis of the ANDROMEDA-SHOCK trial
}

Eduardo Kattan ${ }^{1}$, Gustavo A. Ospina-Tascón ${ }^{2}$, Jean-Louis Teboul ${ }^{3}$, Ricardo Castro ${ }^{1}$, Maurizio Cecconi ${ }^{4}$, Giorgio Ferri ${ }^{5}$, Jan Bakker ${ }^{6,1,7,8}$, Glenn Hernández ${ }^{*}$ and The ANDROMEDA-SHOCK Investigators

\begin{abstract}
Background: Fluid boluses are administered to septic shock patients with the purpose of increasing cardiac output as a means to restore tissue perfusion. Unfortunately, fluid therapy has a narrow therapeutic index, and therefore, several approaches to increase safety have been proposed. Fluid responsiveness (FR) assessment might predict which patients will effectively increase cardiac output after a fluid bolus (FR+), thus preventing potentially harmful fluid administration in non-fluid responsive (FR-) patients. However, there are scarce data on the impact of assessing FR on major outcomes.

The recent ANDROMEDA-SHOCK trial included systematic per-protocol assessment of FR. We performed a post hoc analysis of the study dataset with the aim of exploring the relationship between FR status at baseline, attainment of specific targets, and clinically relevant outcomes.

Methods: ANDROMEDA-SHOCK compared the effect of peripheral perfusion- vs. lactate-targeted resuscitation on 28-day mortality. FR was assessed before each fluid bolus and periodically thereafter. FR+ and FR- subgroups, independent of the original randomization, were compared for fluid administration, achievement of resuscitation targets, vasoactive agents use, and major outcomes such as organ dysfunction and support, length of stay, and 28day mortality.

Results: FR could be determined in 348 patients at baseline. Two hundred and forty-two patients (70\%) were categorized as fluid responders. Both groups achieved comparable successful resuscitation targets, although nonfluid responders received less resuscitation fluids (0 [0-500] vs. 1500 [1000-2500] mL; $p$ 0.0001), exhibited less positive fluid balances, but received more vasopressor testing. No difference in clinically relevant outcomes between FR+ and FR- patients was found, including 24-h SOFA score (9 [5-12] vs. 8 [5-11], $p=0.4)$, need for MV ( $78 \%$ vs. $72 \%, p=0.16$ ), need for RRT ( $18 \%$ vs. $21 \%, p=0.7$ ), ICU-LOS (6 [3-11] vs. 6 [3-16] days, $p=0.2$ ), and 28-day mortality ( $40 \%$ vs. $36 \%, p=0.5)$. Only thirteen patients remained fluid responsive along the intervention period.

Conclusions: Systematic assessment allowed determination of fluid responsiveness status in more than $80 \%$ of patients with early septic shock. Fluid boluses could be stopped in non-fluid responsive patients without any negative impact on clinical relevant outcomes. Our results suggest that fluid resuscitation might be safely guided by FR assessment in septic shock patients.

(Continued on next page)
\end{abstract}

\footnotetext{
* Correspondence: glenn@med.puc.cl; glennguru@gmail.com

${ }^{1}$ Departmento de Medicina Intensiva, Facultad de Medicina, Pontificia

Universidad Católica de Chile, Avenida Diagonal Paraguay 362, Santiago,

Chile

Full list of author information is available at the end of the article
}

(c) The Author(s). 2020 Open Access This article is distributed under the terms of the Creative Commons Attribution 4.0 International License (http://creativecommons.org/licenses/by/4.0/), which permits unrestricted use, distribution, and reproduction in any medium, provided you give appropriate credit to the original author(s) and the source, provide a link to the Creative Commons license, and indicate if changes were made. The Creative Commons Public Domain Dedication waiver (http://creativecommons.org/publicdomain/zero/1.0/) applies to the data made available in this article, unless otherwise stated. 
(Continued from previous page)

Trial registration: ClinicalTrials.gov identifier, NCT03078712. Registered retrospectively on March 13, 2017.

Keywords: Septic shock, Fluid responsiveness, Fluid overload, Early resuscitation,

\section{Background}

Fluid administration is the first line therapy to reverse sepsisinduced tissue hypoperfusion $[1,2]$. For this purpose, fluids are administered either as fluid loading at the emergency department [2], or later as fluid challenges during advanced intensive care unit (ICU)-based resuscitation [3]. However, as any other drug, fluids have a narrow therapeutic index. Insufficient fluid resuscitation may lead to progressive tissue hypoperfusion and organ dysfunction [4], while excess fluids could induce detrimental fluid overload [5-8].

Fluid responsiveness (FR) is a physiologic cardiovascular condition where an increase in preload induced by a fluid bolus leads to an increase in cardiac output (CO) by more than $10-15 \%$ [9-11]. In non-fluid responsive (FR-) patients, fluid administration does not significantly increase $\mathrm{CO}$ and may contribute to congestion and fluid overload. The rationale to assess FR is then to try to optimize fluid resuscitation in critically ill patients by focusing fluid boluses in FR+ hypoperfused patients and by preventing harmful fluid administration in FR- patients.

Multiple tests have been described to assess FR at the bedside [12-15]. They allow to determine the position of the patient's heart on its systolic function curve. By applying the appropriate tests, FR can be assessed in a wide variety of clinical settings [16-18]. However, despite their relative simplicity, lack of cost, and side effects, the use of FR tests has not completely permeated into routine clinical practice $[19,20]$. Moreover, recent major septic shock studies did not include systematic assessment of FR as part of the research protocols [21-25]. Only a few small pilot sepsis studies have tested the impact of FR assessment on major outcomes without conclusive results [26-29]. Indeed, a major problem is that despite a relatively sound physiological background, the concept of FR has not yet demonstrated its usefulness to improve the quality or safety of fluid administration during septic shock resuscitation.

ANDROMEDA-SHOCK is the first major study that incorporated systematic per-protocol assessment of FR [30] and thus provides the opportunity to get insight into the potential clinical relevance of this monitoring. We performed a post hoc analysis of the study dataset with the aim of exploring the relationship between FR status at baseline, fluid administration, attainment of specific targets, and clinically relevant outcomes.

\section{Materials and methods}

The complete protocol, statistical analysis, and main results of the ANDROMEDA-SHOCK trial have been previously published [30-32]. Institutional review boards at each participating center approved the study. Informed consent was obtained directly from the patients or the surrogates.

The ANDROMEDA-SHOCK trial was a prospective, multicenter, parallel-group randomized control trial conducted in 5 Latin-American countries from March 2017 to March 2018, including a total of 424 patients with septic shock. Its main objective was to evaluate the impact on 28-day mortality of a peripheral perfusion (PPTR) - vs. lactate level-targeted resuscitation (LTR) over an 8-h intervention period. Eligible patients were included within a time frame of $4 \mathrm{~h}$ after the diagnosis [28], and they were subjected to a sequential and stepwise resuscitation algorithm aiming to normalize capillary refill time $(\mathrm{CRT},<3 \mathrm{~s})$ vs. to normalize arterial lactate levels $(<2 \mathrm{mmol} / \mathrm{L}$ or at least, $20 \%$ decrease every $2 \mathrm{~h})$.

The goal of fluid resuscitation in the ANDROMEDASHOCK trial was to restore tissue perfusion as represented by CRT or lactate targets. Fluid responsiveness was assessed before each fluid bolus and periodically during the 8-h intervention period. Specific tests were selected according to the particular clinical context and local preferences [31]. A decision algorithm was proposed to guide FR assessment in complex cases (Additional file 1). Only tests with a validated cutoff for a 10$15 \%$ increase in $\mathrm{CO}$ after a fluid challenge were allowed $[13,14,17,33,34]$. A detailed description of each used test including its cutoffs is presented in Additional file 2. Obligatory CO assessment was not part of the protocol, and thus, prediction of FR status was based on previously reported cutoffs for each test.

Fluid resuscitation was indicated and focused on perfusion target achievement. As a general principle, fluid boluses were administered only to patients in a FR+ status as part of protocolized resuscitation aimed at achieving the specific allocated target.

Fluid resuscitation was avoided in patients with a demonstrated FR- status. In these patients, further resuscitation when required was performed applying non-fluidrelated steps of the protocol [31]. Whatever the FR status, further fluid boluses were not administered when perfusion targets were achieved in each group.

The first resuscitation step for $\mathrm{FR}+$ patients in the PPTR group was to administer a fluid bolus of $500 \mathrm{~mL}$ of crystalloids every $30 \mathrm{~min}$ until normalizing CRT. Status of FR and central venous pressure (CVP) were 
reassessed after each fluid bolus, and fluids were stopped before achieving the target if the patient turned FR- or if CVP increased $\geq 5 \mathrm{mmHg}$. In the LTR group, lactate was measured every $2 \mathrm{~h}$ and further fluid resuscitation was decided depending on target achievement. During the 2-h time intervals, 500 - $\mathrm{mL}$ fluid boluses were repeatedly administered every $30 \mathrm{~min}$, provided that the patient did not become FR- or the CVP safety limit was not reached in the meantime.

If patients did not achieve the perfusion target for whatever reason during the fluid resuscitation step, the next protocol interventions were vasopressor or inodilator tests as previously reported [30]. All interventions had predefined safety limits $[31,35]$, including fluid administration in patients in whom FR could not be determined.

\section{Data collection and statistical analysis}

Data for this study were obtained from the original ANDROMEDA-SHOCK trial database. Patients were categorized according to FR status at baseline into three groups: FR+, FR-, and non-assessable. Only patients in whom FR could be determined were considered for further analysis.

The main outcome was 28-day mortality, while clinically relevant secondary outcomes were daily sequential organ failure assessment (SOFA) scores [35], need of mechanical ventilation (MV) and renal replacement therapy (RRT), MV days, and ICU and hospital length of stay (LOS), among others [30].

Demographic and clinical data, including age, comorbidities (Charlson score), severity scores, source, and hemodynamic and perfusion variables, were registered at baseline. All protocol-related procedures and monitoring were recorded during the 8 -h intervention period, including repeated FR assessment, resuscitation and total fluids, and fluid balances. FR was assessed before any fluid bolus, but also at predefined intervals during the intervention period.

After discarding normal distribution, non-parametric tests were selected to determine differences between groups. Descriptive statistics are shown as median [interquartile range] or percentage (\%) accordingly. MannWhitney $U$, Kruskal-Wallis, chi-square, Fisher's exact, and Z-proportion tests, with Bonferroni's post hoc correction, were used when appropriate. Data was analyzed with Minitab v17 (Minitab Inc., State College, PA) and Graphpad Prism (Graphpad Softwares, La Joya, CA) softwares. Two-tailed $p$ value $<0.05$ was considered statistically significant.

\section{Results}

Fluid responsiveness was unavailable in 76 patients at baseline, and this increased to 104 at $8 \mathrm{~h}$. The group of patients categorized as with unavailable FR status over time $(0$ to $8 \mathrm{~h})$ was the sum of early deaths and patients in whom it could be not determined mainly because of technical reasons. FR could be determined in 348 of 424 patients $(82 \%)$ at randomization time. Of this sample, $242(70 \%)$ patients were categorized as fluid responders. These FR+ patients had received a pre-protocol fluid loading of 26.7 [17-40] vs. 26.8 [20-38] $\mathrm{mL} / \mathrm{kg}(p=0.8)$ in FR- patients. Baseline demographic and severity characteristics of groups are shown in Table 1. A description of the whole population including the group of patients in whom FR could not be determined is shown in Additional file 3.

Evolution of perfusion-related parameters during the intervention period for each group is presented in Additional file 4 . No difference in clinically relevant outcomes between FR+ and FR- patients at baseline was observed, including 24-h SOFA score (9 [5-12] vs. 8 [511], $p=0.4$ ), need for MV (78\% vs. $72 \%, p=0.16)$, MV days $(4[2-10]$ vs. 5 [2-14], $p=0.2)$, need for RRT $(18 \%$ vs. $21 \%, p=0.7)$, ICU-LOS (6 [3-11] vs. 6 [3-16] days, $p=0.2$ ), hospital LOS (13 [5-39] vs. 16 [8-28] days, $p=$ 0.2 ), and 28 -day mortality ( $40 \%$ vs. $36 \%, p=0.5$ ).

Achievement of resuscitation targets was comparable between FR+ and FR- subgroups at 2 and $8 \mathrm{~h}$, but the trend was significantly higher for FR+ (Fig. 1). Use of fluids and vasoactive drugs during the study protocol is shown in Table 2. Fluid responders received significantly more fluids and completed the intervention period, with a more positive fluid balance. No statistically significant difference was found in norepinephrine requirements. On the other hand, more patients in the FR- group underwent a vasopressor or an inodilator test. Fluid balance $(2056$ [1012-3746] vs. 1650 [550-2560] $\mathrm{mL} ; p=$ 0.02 ) was also significantly more positive at $24 \mathrm{~h}$ in the $\mathrm{FR}+$ group.

Three hundred and twenty-eight patients were mechanically ventilated at the start of the protocol (77\%). Different tests were used for the assessment of FR as depicted in Table 3. The most commonly used techniques in mechanically ventilated patients were pulse pressure variation (PPV) [36], and passive leg raising (PLR) with pulse pressure (PLR-PP) $[14,16]$ or velocity time integral (PLR-VTI). In non-ventilated patients, the most frequently used tests were PLR-PP and PLR-VTI. Fluid responsiveness results for each test are shown in Additional file 5.

Most patients evolved into a fluid-unresponsive state during the 8-h intervention period (Fig. 2). Of note, less than $15 \%$ of patients became $\mathrm{FR}+$ at any time point in the FR- group, and only 13 patients that were FR+ at baseline maintained this status at the end of the intervention period. 
Table 1 Baseline characteristics of study participants

\begin{tabular}{|c|c|c|c|}
\hline & Fluid responders & Non-fluid responders & $p$ value \\
\hline N & 242 & 106 & \\
\hline Age (years) & $63[50-74]$ & $66[53-75]$ & 0.56 \\
\hline Sex, $N(\%)$ & Female, 115 (48\%) & Female, 51 (48\%) & 0.86 \\
\hline \multirow[t]{2}{*}{ Study arm, N (\%) } & LTR, 115 (48\%) & LTR, 57 (54\%) & \multirow[t]{2}{*}{0.3} \\
\hline & PPTR, 127 (52\%) & PPTR, 49 (46\%) & \\
\hline APACHE score & 23 [18-29] & $21[15-27]$ & 0.09 \\
\hline SOFA score & $10[7-12]$ & $9[7-12]$ & 0.52 \\
\hline Charlson index & $3[1-5]$ & $3[1-5]$ & 0.4 \\
\hline \multirow[t]{4}{*}{ Sepsis origin, N (\%) } & Abdominal, 92 (38\%) & Abdominal, 34 (32\%) & \multirow[t]{4}{*}{0.3} \\
\hline & Pulmonary, 71 (29\%) & Pulmonary, 25 (24\%) & \\
\hline & Urinary, 53 (22\%) & Urinary, 24 (23\%) & \\
\hline & Other, 26 (11\%) & Other, 23 (21\%) & \\
\hline MAP (mmHg) & $66[60-75]$ & 67 [62-78] & 0.2 \\
\hline Pulse pressure (mmHg) & 45 [35-59] & 46 [33-59] & 0.76 \\
\hline DBP (mmhg) & $51[45-59]$ & 52 [44-59] & 0.5 \\
\hline CVP $(m m H g)$ & $9[5-12]$ & $10[7-14]$ & 0.001 \\
\hline Pre-protocol fluids (mL/kg) & $26.7[17-40]$ & $26.8[20-38]$ & 0.8 \\
\hline Norepinephrine dose (mcg/kg/min) & $0.22[0.1-0.4]$ & $0.21[0.12-0.4]$ & 0.8 \\
\hline Arterial lactate (mmol/L) & $3.8[2.8-5.5]$ & $3.6[2.8-5.5]$ & 0.4 \\
\hline CRT (s) & $5[4-6]$ & $4[3-6]$ & 0.002 \\
\hline $\mathrm{ScvO}_{2}(\%)$ & $72[63-78]$ & $74[65-81]$ & 0.27 \\
\hline Delta $\mathrm{pCO}_{2}(\mathrm{v}-\mathrm{a})$ & $7[5-10]$ & $7[5-10]$ & 0.57 \\
\hline
\end{tabular}

Tests: Mann-Whitney or Fisher's exact test, accordingly

LTR lactate level-targeted group, PPTR peripheral perfusion-targeted group, APACHE II Acute Physiology And Chronic Health Evaluation II, SOFA sequential organ failure assessment score, MAP mean arterial pressure, $D B P$ diastolic blood pressure, $C V P$ central venous pressure, $C R T$ capillary refill time, $S c v O_{2}$ central venous oxygen saturation, Delta $\mathrm{pCO}_{2}(\mathrm{v}-a)$ difference between central venous carbon dioxide pressure and arterial carbon dioxide pressure

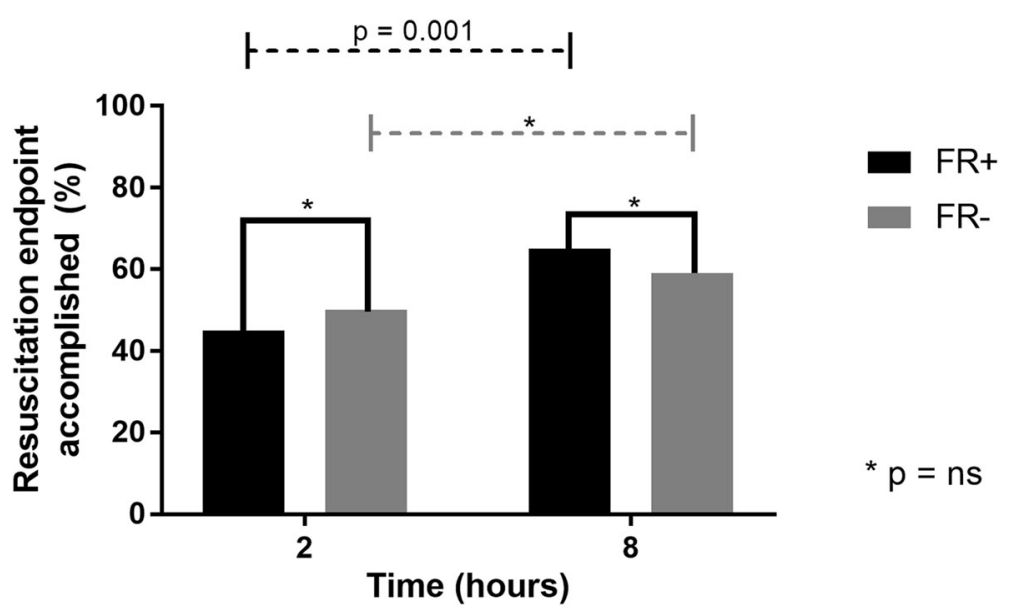

Fig. 1 Achievement of resuscitation endpoints during the intervention period according to fluid responsiveness status at baseline. FR+, fluid responsive; FR-, non-fluid responsive 
Table 2 Resuscitation therapies during the 8-h intervention period

\begin{tabular}{llll}
\hline & Fluid responders & Non-fluid responders & $p$ value \\
\hline Fluids administered pre-protocol $(\mathrm{mL})$ & $2000[1194-2643]$ & $2000[1200-2500]$ & 0.86 \\
FR assessments performed $(\mathrm{N})$ & $8[7-10]$ & $5[5-6]$ & $0[0-0]$ \\
Fluid bolus 0-2 $\mathrm{h}(\mathrm{mL})$ & $1000[500-1500]$ & $0[0-500]$ & 0.03 \\
Fluid bolus 0-8 $\mathrm{h}(\mathrm{mL})$ & $1500[1000-2500]$ & $1748[1090-2881]$ & 0.0001 \\
Total fluids 0-8 $\mathrm{h}(\mathrm{mL})$ & $2500[1594-3840]$ & $1244[395-2251]$ & 0.0001 \\
Fluid balance $8 \mathrm{~h}(\mathrm{~mL})$ & $1672[894-2842]$ & $0.21[0.12-0.4]$ & 0.003 \\
Norepinephrine dose $0 \mathrm{~h}(\mathrm{mcg} / \mathrm{kg} / \mathrm{min})$ & $0.22[0.1-0.4]$ & $0.23[0.11-0.4]$ & 0.006 \\
Norepinephrine dose $2 \mathrm{~h}(\mathrm{mcg} / \mathrm{kg} / \mathrm{min})$ & $0.22[0.09-0.45]$ & $0.20[0.1-0.45]$ & 0.8 \\
Norepinephrine dose $4 \mathrm{~h}(\mathrm{mcg} / \mathrm{kg} / \mathrm{min})$ & $0.24[0.1-0.42]$ & $0.16[0.08-0.4]$ & 0.5 \\
Norepinephrine dose $8 \mathrm{~h}(\mathrm{mcg} / \mathrm{kg} / \mathrm{min})$ & $0.24[0.1-0.45]$ & $46 / 106(43.3 \%)$ & 0.9 \\
Vasopressor test $(\%)$ & $74 / 242(30.5 \%)$ & $23 / 106(21.6 \%)$ & 0.3 \\
Inodilator test $(\%)$ & $33 / 242(13.6 \%)$ & 0.02 \\
\hline
\end{tabular}

Tests: Mann Whitney or Fisher's exact test, accordingly

$F R$ fluid responsiveness

\section{Discussion}

Our main findings can be summarized as follows: (a) fluid responsiveness status could be determined in $82 \%$ of early septic shock patients by using diverse tests depending on the clinical context; (b) $30 \%$ of patients were already non-fluid responsive before starting ICUbased resuscitation; and (c) despite receiving less fluids, non-fluid responders at baseline resolved hypoperfusion in a similar proportion than FR+ patients by following other steps of the protocol with no difference in clinically relevant outcomes. Our results do not support a clear benefit of FR assessment on major outcomes but suggest that withholding fluid boluses in FR- patients appears to be safe.

Fluid overload has been associated with increased mortality and morbidity in patients with septic shock. Therefore, several strategies to deliver fluid resuscitation in a more physiologic, rational, and restrictive way are being tested in ongoing trials [25, 37]. Among these strategies, early use of vasopressor support [24, 38], and selecting more flow-sensitive and rapid-response targets [30], could be promising. Systematic assessment of FR might aid in preventing unnecessary fluid administration in FR- patients. However, one major concern could be the potential harm of restricting fluids during septic shock resuscitation. The $30 \%$ of patients who were FR- at baseline received around $1000 \mathrm{~mL}$ less fluids than $\mathrm{FR}+$ patients during the first $2 \mathrm{~h}$ and $1500 \mathrm{~mL}$ less during the overall 8-h intervention period. Remarkably, they achieved resuscitation targets in the same proportion as FR+ patients, and exhibited comparable mortality and organ dysfunction improvement. Thus, these data suggest that stopping fluid resuscitation in FR- patients appears to be safe. Nevertheless, FR- patients were more frequently subjected to a protocolized vasopressor test to increase mean arterial pressure target in previously hypertensive patients, and/or to inodilators aimed at resolving hypoperfusion instead of insisting on fluid administration.

Table 3 Techniques used to assess fluid responsiveness at baseline

\begin{tabular}{|c|c|c|c|}
\hline Technique & $\begin{array}{l}\text { Peripheral perfusion-targeted resuscitation ( } n= \\
\text { 212) }\end{array}$ & $\begin{array}{l}\text { Lactate-targeted resuscitation }(n= \\
212)\end{array}$ & Total \\
\hline \multicolumn{4}{|l|}{ Fluid responsiveness } \\
\hline Undetermined & $36(17 \%)$ & $40(18.9 \%)$ & $76(18 \%)$ \\
\hline Pulse pressure variation & $73(34.4 \%)$ & $71(33.5 \%)$ & $\begin{array}{l}144 \\
(33.9 \%)\end{array}$ \\
\hline $\begin{array}{l}\text { Passive leg rising assessed using PP, CO, or } \\
\text { VTI }\end{array}$ & $70(33.0 \%)$ & $74(34.9 \%)$ & $\begin{array}{l}144 \\
(33.9 \%)\end{array}$ \\
\hline End-expiratory occlusion test & $3(1.4 \%)$ & $4(1.9 \%)$ & $7(1.7 \%)$ \\
\hline Inferior vena cava variation & $24(11.4 \%)$ & $17(8.0 \%)$ & $41(9.7 \%)$ \\
\hline Stroke volume variation & $6(2.8 \%)$ & $6(2.8 \%)$ & $12(2.8 \%)$ \\
\hline
\end{tabular}

$P P$ pulse pressure, $C O$ cardiac output, VTI velocity time integral 


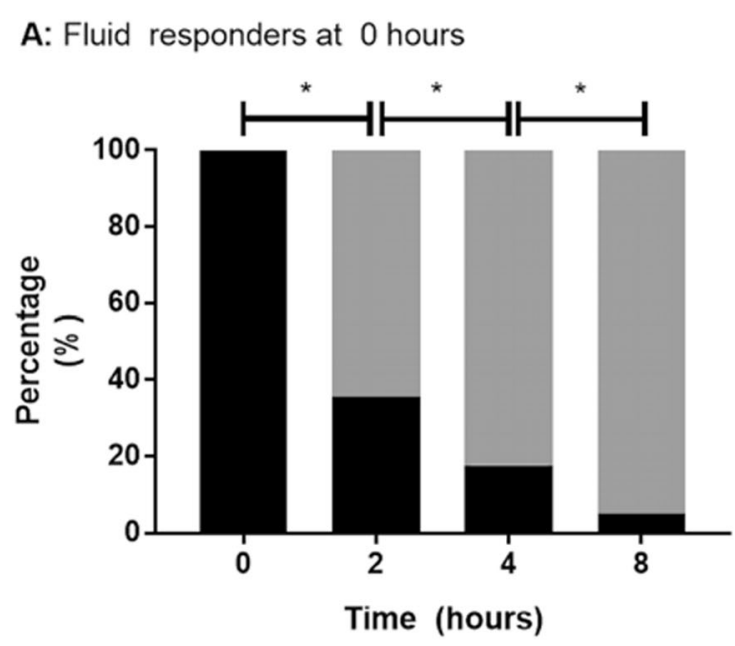

Fluid responsiveness

Non-fluid responsiveness

B: Non-fluid responders at 0 hours

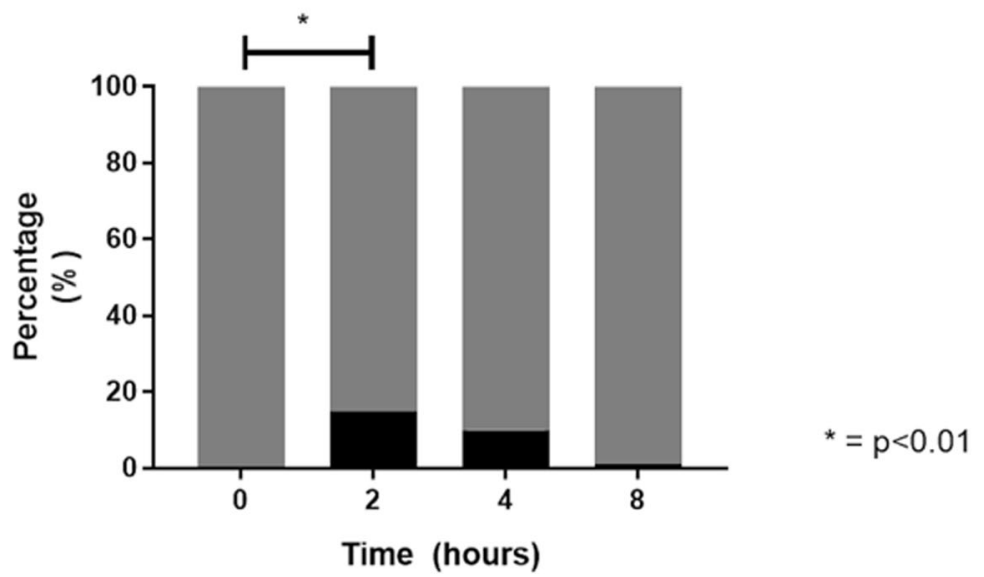

Fig. 2 a, b Evolution of fluid responsiveness during protocolized resuscitation, according to fluid responsiveness status at baseline

Although there is an extensive literature on the fluid responsiveness concept and background physiology, as well as on assessment techniques, the use of FR tests is not considered as a standard of care, nor has been included in recent septic shock trials. There are many possible explanations for this fact. First, some of these techniques are time-consuming, operator-dependent, not universally applicable, and with many inherent limitations. Second, there is a gray zone around the published cutoff values that somehow turns decision-making on further fluid resuscitation uncertain and complex [9]. Third, there are many misconceptions on the subject, particularly the erroneous idea that turning patients into a fluid-unresponsive state, which by definition is physiologically abnormal, is a valid objective. Therefore, the use of FR assessment could paradoxically lead to a potential fluid overload instead of preventing it. Fourth, the relevance of FR assessment for guiding therapy or the impact on major outcomes has not been demonstrated. Only 4 small randomized controlled studies including a total of 365 septic patients [26-29, 39] compared fluid responsiveness-guided resuscitation to standard techniques, and found no significant difference in major or secondary outcomes. However, the studies involved highly heterogeneous cohorts of patients and settings, and were probably underpowered to detect real differences. In this sense, although this study did not demonstrate outcome differences, it supports the idea that restricting fluid boluses in FR- septic shock patients appears at least to be safe. Future appropriately powered studies, and eventually with a randomized controlled design, should determine the definitive role of systematic FR assessment in septic shock resuscitation strategies.

The behavior of FR status during the 8-h intervention period in our cohort is intriguing. The effect of fluid boluses on $\mathrm{CO}$ and fluid responsiveness is thought to be 
transient based on previous pharmacodynamic studies [40]. This has been attributed to capillary leakage or blood redistribution from stressed to unstressed volumes $[3,9]$. In our study, we did not assess CO systematically, but found that the FR+ status disappeared in almost all patients after receiving a median of only $1500 \mathrm{~mL}$ during the intervention period. We do not have an explanation for this finding, but the fact that only $40 \%$ of the initial $\mathrm{FR}+$ patients were still fluid responsive $2 \mathrm{~h}$ after inclusion reinforces the idea that FR should be periodically reassessed when performing an active septic shock resuscitation [41]. On the other hand, it is unclear why so many patients exhibited a FR- state so early during resuscitation. Pre-ICU fluid loading might be responsible for this observation, but the amount of fluids received was within the limits of current recommendations. Unfortunately, the diastolic and systolic cardiac functions were not systematically evaluated whereby we cannot rule out the presence of sepsis-induced myocardial dysfunction or even previous cardiomyopathy.

This study presents several limitations. First, it has the inherent limitations of a post hoc analysis, so conclusions should be considered only as hypothesisgenerating. Second, the use of some techniques might be criticized. PPV was used in one third of the patients, which is far more than expected considering the numerous limitations of this method [12]. On the other hand and despite current recommendations [42], PLR-PP was used more frequently than PLR-VTI to assess FR in spontaneous breathing patients. This could be also criticized since the changes in pulse pressure during PLR have a low sensitivity although good specificity to assess FR [9]. Indeed, a positive test (increase in PP during PLR) is reliable for detecting a FR+ state, but a negative test is not. Some centers preferred to start with PLR-PP which is much faster and easier to be applied on a 24/7 basis especially in resource-constrained settings. Anyway, the fact that the proportion of FR+ versus FR- was relatively comparable whatever the test used tends to support its use (Additional file 5). In addition, a recent retrospective study including 491 patients showed that changes in $\mathrm{CO}$ can be roughly predicted by increases in PP [43]. Third, our protocol did not mandate advanced hemodynamic monitoring, and therefore, data on cardiac output or stroke volume are lacking. Therefore, we acknowledge that classifying patients according to FR status might have some inherent bias since it was not confirmed by direct $\mathrm{CO}$ measurement or an effective fluid challenge in most of the patients. However, direct measurements of $\mathrm{CO}$ are not always available in clinical practice [43] and this is one of the general limitations of FR assessment techniques. Fourth, FR could not be determined in $18 \%$ of the patients, mainly because of logistic reasons. Fifth, we cannot support the external validity of our results, since only centers with experience in FR assessment were included. Sixth, we did not assess criteria of fluid overload. So, we cannot ensure that restricting fluid boluses in FR- patients prevented this complication. Despite all these limitations, it is encouraging that systematic assessment of FR was feasible in a context of mostly public hospitals in medium-income countries, and at least, this allowed to safely avoid potentially harmful fluid resuscitation in almost one third of septic shock patients.

\section{Conclusions}

Systematic assessment allowed determination of fluid responsiveness status in more than $80 \%$ of patients with early septic shock. Fluid boluses could be stopped in non-fluid responsive patients without any negative impact on clinical relevant outcomes. Our results suggest that fluid resuscitation might be safely guided by FR assessment in septic shock patients.

\section{Supplementary information}

Supplementary information accompanies this paper at https://doi.org/10. 1186/s13054-020-2732-y.

\begin{abstract}
Additional file 1: Fluid responsiveness assessment algorithm. PPV: Pulse pressure variation; PLR-VTI: Passive leg raising assessed using velocity time integral; IVCV: Inferior vena cava variation; SVCV: Superior vena cava variation; EEOT: end-expiratory occlusion test; SW: stroke volume variation; ARDS: Acute respiratory distress syndrome; CO: Cardiac output; Cl: Cardiac index; VTI: Velocity time integral; Vt: Tidal volume; PBW: Predicted body weight.
\end{abstract}

Additional file 2: Technical details of different fluid responsiveness assessment techniques.

Additional file 3: Baseline characteristics including the subgroup of patients in whom FR could not be assessed.

Additional file 4: Evolution of perfusion parameters during the first $24 \mathrm{~h}$.

Additional file 5: Distribution of fluid responsiveness at baseline according to the test used.

\section{Abbreviations}

APACHE II: Acute Physiology And Chronic Health Evaluation II; ARDS: Acute respiratory distress syndrome; CO: Cardiac output; CRT: Capillary refill time; CVP: Central venous pressure; DBP: Diastolic blood pressure; Delta pCO2(va): Difference between central venous carbon dioxide pressure and arterial carbon dioxide pressure; EEOT: End-expiratory occlusion test; FR: Fluid responsiveness; FR-: Non-fluid responsive; FR+: Fluid responsive;

ICU: Intensive care unit; IVCV: Inferior vena cava variation; LOS: Length of stay; MAP: Mean arterial pressure; MV: Mechanical ventilation; PLR: Passive leg raising; PLR-PP: Passive leg raising assessed using pulse pressure; PLRVTI: Passive leg raising assessed using velocity time integral; PPV: Pulse pressure variation; RRT: Renal replacement therapy; ScvO2: Central venous oxygen saturation; SOFA: Sequential organ failure assessment; SSC: Surviving Sepsis Campaign; SW: Stroke volume variation

\section{Acknowledgments}

The ANDROMEDA-SHOCK and the Latin America Intensive Care Network (LIVEN) Investigators are listed below.

Writing and Steering Committee: Glenn Hernandez (co-chair), Gustavo

Ospina-Tascón, Lucas Petri Damiani, Elisa Estenssoro, Arnaldo Dubin, Javier Hurtado, Gilberto Friedman, Ricardo Castro, Leyla Alegría, Jean-Louis Teboul, Maurizio Cecconi, Giorgio Ferri, Manuel Jibaja, Ronald Pairumani, Paula 
Fernández, Diego Barahona, Alexandre Biasi Cavalcanti, and Jan Bakker (cochair).

Study Coordinating Center: Glenn Hernandez, Leyla Alegría, Giorgio Ferri, Nicolás Rodriguez, Patricia Holger, Natalia Soto, Mario Pozo, and Jan Bakker. Data Safety Monitoring Committee: Deborah Cook, Jean-Louis Vincent, Andrew, Rhodes, Bryan P. Kavanagh, Phil Dellinger, and Wim Rietdijk (statistician)

Latin America Intensive Care Network (LIVEN)

Chile: Pontificia Universidad Católica de Chile, Santiago (David Carpio, Nicolás Pavéz, Elizabeth Henriquez, Sebastian Bravo, Emilio Daniel Valenzuela, Magdalena Vera, Jorge Dreyse, Vanessa Oviedo); Hospital Barros-Luco Trudeau, Santiago (Maria Alicia Cid, Macarena Larroulet, Edward Petruska, Claudio Sarabia); Hospital San Juan de Dios, Santiago (David Gallardo, Juan Eduardo Sanchez, Hugo González, José Miguel Arancibia, Alex Muñoz, Germán Ramirez, Florencia Aravena); Hospital Dr. Sótero del Río, Santiago (Andrés Aquevedo, Fabián Zambrano); Hospital Del Salvador, Santiago (Milan Bozinovic, Felipe Valle, Manuel Ramirez, Victor Rossel, Pilar Muñoz, Carolina Ceballos); Hospital Herminda Martinez, Chillán (Christian Esveile, Cristian Carmona, Eva Candia, Daniela Mendoza); Hospital San Juan de Dios, Curicó (Aída Sanchez); Hospital Guillermo Grant Benavente, Concepción (Daniela Ponce, Jaime Lastra, Bárbara Nahuelpán, Fabrizio Fasce); Hospital Clínico Universidad de Chile, Santiago (Cecilia Luengo, Nicolas Medel, Cesar Cortés). Argentina: Clínica La Pequeña Familia, Junín (Luz Campassi); Sanatorio Otamendi y Miroli, Buenos Aires (Paolo Rubatto, Brenda Nahime Horna, Mariano Furche); Hospital Provincial del Centenario, Rosario (Juan Carlos Pendino, Lisandro Bettini); Sanatorio Parque, Rosario (Carlos Lovesio, María Cecilia González, Jésica Rodruquez); Hospital Interzonal San Martín, La Plata (Héctor Canales, Francisco Caminos); Sanatorio Allende Nueva Córdoba (Cayetano Galletti, Estefanía Minoldo, María José Aramburu, Daniela Olmos); Uruguay: Hospital Español Juan J Crottogini, Montevideo (Nicolás Nin, Jordán Tenzi, Carlos Quiroga, Pablo Lacuesta, Agustín Gaudín, Richard Pais, Ana Silvestre, Germán Olivera); Hospital de Clínicas, Montevideo (Gloria Rieppi, Dolores Berrutti). Ecuador: Hospital Universitario del Rio, Cuenca (Marcelo Ochoa, Paul Cobos, Fernando Vintimilla); Hospital Eugenio Espejo, Quito (Vanessa Ramirez, Milton Tobar, Fernanda García, Fabricio Picoita, Nelson Remache); Hospital San Francisco, Quito (Vladimir Granda, Fernando Paredes, Eduardo Barzallo, Paul Garcés); Hospital Carlos Andrade Marín, Quito (Fausto Guerrero, Santiago Salazar, German Torres, Cristian Tana, José Calahorrano, Freddy Solis); Hospital IESS, Ibarra (Pedro Torres, Luís Herrera, Antonio Ornes, Verónica Peréz, Glenda Delgado, Alexei López, Eliana Espinosa, José Moreira); Hospital General Docente Calderón, Quito (Blanca Salcedo, Ivonne Villacres, Jhonny Suing, Marco Lopez, Luis Gomez, Guillermo Toctaquiza, Mario Cadena Zapata, Milton Alonso Orazabal, Ruben Pardo Espejo, Jorge Jimenez, Alexander Calderón); Hospital Enrique Garcés, Quito (Gustavo Paredes, José Luis Barberán, Tatiana Moya). Colombia: Hospital San Vicente de Paul, Medellín (Horacio Atehortua, Rodolfo Sabogal); Hospital de Santa Clara, Bogotá (Guillermo Ortiz, Antonio Lara); Hospital Universitario de Ñarino E.S.E, Pasto (Fabio Sanchez, Alvaro Hernán Portilla, Humberto Dávila, Jorge Antonio Mora); Fundación Valle del Lili, Cali (Luis Eduardo Calderón, Ingrid Alvarez, Elena Escobar, Alejandro Bejarano, Luis Alfonso Bustamante, José Luis Aldana).

\section{Authors' contributions}

EK, GAO-T, JB, JLT, RC, GF, MC, and GH contributed to the study conception, design, and manuscript preparation. GF was involved in the data collection. EK and RC performed the statistical analyses. All authors read and approved the final manuscript.

\section{Funding}

The study was supported in part by grant FONDECYT (Chile) 1170043, and some logistical support from the Departamento de Medicina Intensiva of the Pontificia Universidad Católica de Chile.

\section{Availability of data and materials}

The datasets generated and/or analyzed during the current study are not publicly available until February 2020 when we expect to have published all sub-studies, but are available before from the corresponding author on reasonable request.

\section{Ethics approval and consent to participate}

The study was approved by institutional review boards at each of the 28 study sites. Informed consent was obtained from patients or legally authorized surrogates.
The first and main institutional review board was Comité Ético-Científico, Facultad de Medicina, Pontificia Universidad Católica de Chile, March 2017. ID 161012007.

\section{Consent for publication}

Not applicable

\section{Competing interests}

The authors declare that they have no competing interests.

\section{Author details}

'Departmento de Medicina Intensiva, Facultad de Medicina, Pontificia Universidad Católica de Chile, Avenida Diagonal Paraguay 362, Santiago, Chile. ${ }^{2}$ Department of Intensive Care Medicine, Fundación Valle del Lili, Universidad ICESI, Cali, Colombia. ${ }^{3}$ Service de réanimation médicale, Hopital Bicetre, Hopitaux Universitaires Paris-Sud; Assistance Publique Hôpitaux de Paris, Université Paris-Sud, Paris, France. ${ }^{4}$ Department of Biomedical Sciences, Humanitas Clinical and Research Center, Humanitas University, Milan, Italy. ${ }^{5}$ Unidad de Cuidados Intensivos, Hospital Barros Luco Trudeau, Santiago, Chile. ${ }^{6}$ Department of Intensive Care Adults, Erasmus MC University Medical Center, Rotterdam, Netherlands. ${ }^{7}$ Department of Pulmonary and Critical Care, New York University, New York, USA. ${ }^{8}$ Division of Pulmonary, Allergy, and Critical Care Medicine, Columbia University Medical Center, New York, USA.

\section{Received: 20 August 2019 Accepted: 10 January 2020}

Published online: 23 January 2020

\section{References}

1. Singer M, Deustchman C, Warren Seymour C, Shankar-Hari M, Annane D, Bauer $\mathrm{M}$, et al. The third international consensus definitions for sepsis and septic shock (Sepsis-3). JAMA. 2016;315:801-10.

2. Rhodes A, Evans L, Alhazzani W, Levy M, Antonelli M, Ferrer R, et al. Surviving Sepsis Campaign: international guidelines for management of sepsis and septic shock: 2016. Crit Care Med. 2017;45:486-552.

3. Malbrain MLNG, Van Regenmortel N, Saugel B, De Tavernier B, Van Gaal PJ, Joannes-Boyau O, et al. Principles of fluid management and stewardship in septic shock: it is time to consider the four D's and the four phases of fluid therapy. Ann Intensive Care. 2018;8:66.

4. Monnet $X$, Teboul J-L. My patient has received fluid. How to assess its efficacy and side effects? Ann Intensive Care. 2018;8:54

5. Silversides JA, Perner A, Malbrain MLNG. Liberal versus restrictive fluid therapy in critically ill patients. Intensive Care Med. 2019;45:1440-2.

6. Boyd JH, Forbes J, Nakada TA, Walley KR, Russell JA. Fluid resuscitation in septic shock: a positive fluid balance and elevated central venous pressure are associated with increased mortality. Crit Care Med. 2011;39:259-65.

7. Bagshaw SM, Brophy PD, Cruz D, Ronco C. Fluid balance as a biomarker : impact of fluid overload on outcome in critically ill patients with acute kidney injury. Crit Care. 2008;12:1-3.

8. Wiedemann PW, Bernard A, et al. Comparison of two fluid-management strategies in acute lung injury. NEJM. 2006;354:2564-75.

9. Monnet X, Marik PE, Teboul JL. Prediction of fluid responsiveness : an update. Ann Intensive Care. 2016;6:111.

10. Michard F, Teboul J-L. Predicting fluid responsiveness in ICU patients. Chest. 2002;121:2000-8.

11. Marik PE, Lemson J. Fluid responsiveness: an evolution of our understanding. Br J Anaesth. 2014;112:617-20.

12. Teboul JL, Monnet $X$, Chemla D, Michard F. Arterial pulse pressure variation with mechanical ventilation. Am J Respir Crit Care Med. 2019;199:22-31.

13. Monnet X, Osman D, Ridel C, Lamia B, Richard C, Teboul JL. Predicting volume responsiveness by using the end-expiratory occlusion in mechanically ventilated intensive care unit patients. Crit Care Med. 2009;37:951-6.

14. Monnet $X$, Marik P, Teboul JL. Passive leg raising for predicting fluid responsiveness: a systematic review and meta-analysis. Intensive Care Med. 2016:42:1935-47.

15. Mandeville JC, Colebourn CL. Can transthoracic echocardiography be used to predict fluid responsiveness in the critically ill patient? A systematic review. Crit Care Res Pract. 2012;2012:1-9.

16. Teboul J, Monnet X. Prediction of volume responsiveness in critically ill patients with spontaneous breathing activity. Curr Opin Crit Care. 2008;14: 334-9. 
17. Bortolotti P, Colling D, Colas V, Voisin B, Dewavrin F, Poissy J, et al. Respiratory changes of the inferior vena cava diameter predict fluid responsiveness in spontaneously breathing patients with cardiac arrhythmias. Ann Intensive Care. 2018:8:79.

18. Monnet X, Bleibtreu A, Ferré A, Dres M, Gharbi R, Richard C, et al. Passive leg-raising and end-expiratory occlusion tests perform better than pulse pressure variation in patients with low respiratory system compliance. Crit Care Med. 2012:40:152-7.

19. Cecconi M, Hofer C, Teboul JL, Pettila V, Wilkman E, Molnar Z, et al. Fluid challenges in intensive care: the FENICE study: a global inception cohort study. Intensive Care Med. 2015;41:1529-37.

20. Preau S, Dewavrin F, Demaeght V, Chiche A, Voisin B, Minacori F, et al. The use of static and dynamic haemodynamic parameters before volume expansion: a prospective observational study in six French intensive care units. Anaesth Crit Care Pain Med. 2016;35:93-102.

21. Mouncey PR, Osborn TM, Power GS, Harrison DA, Sadique MZ, Grieve RD, et al. Trial of early, goal-directed resuscitation for septic shock. N Engl J Med. 2015;372:1301-11.

22. Yealy DM, Kellum JA, Huang DT, Barnato AE, Weissfeld LA, Pike F, et al. A randomized trial of protocol-based care for early septic shock. N Engl J Med. 2014;370:1683-93.

23. Peake SL, Delaney A, Bailey M, Bellomo R, Cameron PA, Cooper DJ, et al. Goal-directed resuscitation for patients with early septic shock. N Engl J Med. 2014;371:1496-506.

24. Permpikul C, Tongyoo S, Viarasilpa T, Trainarongsakul T, Chakorn T, Udompanturak S. Early use of norepinephrine in septic shock resuscitation (CENSER). A randomized trial. Am J Respir Crit Care Med. 2019;199:1097-105.

25. Corl KA, Prodromou M, Merchant RC, Gareen I, Marks S, Banerjee D, et al. The Restrictive IV Fluid Trial in Severe Sepsis and Septic Shock (RIFTS): A Randomized Pilot Study. Crit Care Med. 2019:47:951-9.

26. Richard JC, Bayle F, Bourdin G, Leray V, Debord S, Delannoy B, et al. Preload dependence indices to titrate volume expansion during septic shock: a randomized controlled trial. Crit Care. 2015;19:1-13.

27. Kuan W, Sen II, BSH L, Jain S, Lu Q, Cheung YB, et al. Emergency department management of sepsis patients: a randomized, goal-oriented, noninvasive sepsis trial. Ann Emerg Med. 2016:67:367-378.e3.

28. Chen C, Kollef MH. Targeted fluid minimization following initial resuscitation in septic shock. Chest. 2015;148:1462-9.

29. Juneja D, Javeri Y, Bajaj P, Gupta C, Arora V, Malhorta N, et al. Use of stroke volume variation to guide fluid therapy in septic shock for prevention of acute kidney injury. Intensive Care Med. 2009;35:S31.

30. Hernandez G, Ospina-Tascon G, Petri Damiani L, Estenssoro E, Dubin A, Hurtado J, et al. Effect of a resuscitation strategy targeting peripheral perfusion status vs serum lactate levels on 28-day mortality among patients with septic shock. The ANDROMEDA-SHOCK randomized clinical trial. JAMA. 2019;321:654-64.

31. Hernández G, Cavalcanti AB, Ospina-Tascón G, Zampieri FG, Dubin A, Hurtado FJ, et al. Early goal-directed therapy using a physiological holistic view: the ANDROMEDA-SHOCK - a randomized controlled trial. Ann Intensive Care. 2018:8:52

32. Hernández G, Cavalcanti AB, Ospina-Tascón G, Dubin A, Hurtado FJ, Damiani LP, et al. Statistical analysis plan for early goal-directed therapy using a physiological holistic view - the andromeda-shock: a randomized controlled trial. Rev Bras Ter Intensiva. 2018;30:253-63.

33. Marik PE, Cavallazzi R, Vasu T, Hirani A. Dynamic changes in arterial waveform derived variables and fluid responsiveness in mechanically ventilated patients: a systematic review of the literature. Crit Care Med. 2009;37:2642-7

34. Huang $H$, Shen $Q$, Liu $Y, X u H$, Fang $Y$. Value of variation index of inferior vena cava diameter in predicting fluid responsiveness in patients with circulatory shock receiving mechanical ventilation: a systematic review and meta-analysis. Crit Care. 2018;22:1-7.

35. De Backer $D$, Vincent $J$. Should we measure the central venous pressure to guide fluid management? Ten answers to 10 questions. Crit Care. 2018;22:43.

36. Myatra SN, Prabu NR, Dlvatia JV, Monnet X, Kulkarni AP, Teboul JL. The changes in pulse pressure variation or stroke volume variation after a "tidal volume challenge" reliably predict fluid responsiveness during low tidal volume ventilation. Crit Care Med. 2017:45:415-21.

37. Self WH, Semler MW, Bellomo R, Steingrub JS, Talmor D, Yealy DM, et al. Liberal versus restrictive intravenous fluid therapy for early septic shock rationale for a randomized trial. Ann Emerg Med. 2018;72:457-66.
38. Bai X, Yu W, Ji W, Lin Z, Tan S, Duan K, et al. Early versus delayed administration of norepinephrine in patients with septic shock. Crit Care. 2014;18:1-8

39. Ehrman RR, Gallien JZ, Smith RK, Akers KG, Malik AN, Harrison NE, et al. Resuscitation guided by volume responsiveness does not reduce mortality in sepsis. Crit Care Explor. 2019;1:e0015.

40. Aya HD, Ster IC, Fletcher N, Grounds RM, Rhodes A, Cecconi M. Pharmacodynamic analysis of a fluid challenge. Crit Care Med. 2016;44:880-91.

41. Roger C, Zieleskiewicz L, Demattei C, Lakhal K, Piton G, Louart B, et al. Time course of fluid responsiveness in sepsis : the fluid challenge revisiting ( FCREV ) study. Crit Care. 2019;23:179.

42. Monnet $X$, Teboul JL. Assessment of fluid responsiveness: recent advances. Curr Opin Crit Care. 2018;24:190-5.

43. Ait-Hamou Z, Teboul JL, Anguel N, Monnet X. How to detect a positive response to a fluid bolus when cardiac output is not measured? Ann Intensive Care. 2019;9:138.

\section{Publisher's Note}

Springer Nature remains neutral with regard to jurisdictional claims in published maps and institutional affiliations.

\section{Ready to submit your research? Choose BMC and benefit from:}

- fast, convenient online submission

- thorough peer review by experienced researchers in your field

- rapid publication on acceptance

- support for research data, including large and complex data types

- gold Open Access which fosters wider collaboration and increased citations

- maximum visibility for your research: over $100 \mathrm{M}$ website views per year

At BMC, research is always in progress.

Learn more biomedcentral.com/submissions 